\title{
Physical Quantity Representation Data Type
}

National Cancer Institute

\section{Source}

National Cancer Institute. Physical Quantity Representation Data Type. NCI Thesaurus. Code C95674.

A data type comprised of the amount or total number of a measured entity with units that come from a coding system. 